\title{
Pathological complete response after neoadjuvant treatment determines survival in esophageal squamous cell carcinoma patients (NEOCRTEC5010)
}

\begin{abstract}
Jianfei Shen $^{1,2 \#}$, Min Kong ${ }^{1,2 \#}$, Hong Yang ${ }^{3 \#}$, Ke Jin ${ }^{1,2 \#}$, Yuping Chen ${ }^{4 \#}$, Wentao Fang ${ }^{5 \#}$, Zhentao Yu ${ }^{6}$, Weimin $\mathrm{Mao}^{7}$, Jiaqing Xiang ${ }^{8}$, Yongtao Han', Zhijian Chen ${ }^{4,10}$, Haihua Yang ${ }^{11}$, Jiaming Wang ${ }^{5}$, Qingsong Pang ${ }^{6}$, Xiao Zheng ${ }^{7}$, Huanjun Yang ${ }^{8}$, Tao $\mathrm{Li}^{9}$, Xu Zhang ${ }^{3}$, Qun $\mathrm{Li}^{3}$, Geng Wang ${ }^{4}$, Teng Mao , Xufeng Guo ${ }^{5}$, Ting Lin $^{3}$, Mengzhong Liu ${ }^{3}$, Dehua Ma ${ }^{1,2}$, Minhua Ye ${ }^{1,2}$, Chunguo Wang ${ }^{1,2}$, Zheng Wang ${ }^{1,2}$, Alessandro Brunelli ${ }^{12}$, Robert J. Cerfolio ${ }^{13}$, Xavier Benoit D'Journo ${ }^{14}$, Hiran C. Fernando ${ }^{15}$, Florian Lordick ${ }^{16}$, Jianhua Fu ${ }^{3}$, Baofu Chen ${ }^{1,2 *}$, Chengchu Zhu ${ }^{1,2 *}$; written on behalf of the AME Thoracic Surgery Collaborative Group
\end{abstract}

${ }^{1}$ Key Laboratory of Minimally Invasive Techniques \& Rapid Rehabilitation of Digestive System Tumor of Zhejiang Province, Linhai, China; ${ }^{2}$ Department of Cardiothoracic Surgery, Taizhou Hospital of Zhejiang Province Affiliated to Wenzhou Medical University, Linhai, China; ${ }^{3}$ Sun Yat-sen University Cancer Center, State Key Laboratory of Oncology in South China, Guangdong Esophageal Cancer Institute, Collaborative Innovation Center for Cancer Medicine, Guangzhou, China; ${ }^{4}$ Department of Thoracic Surgery, Cancer Hospital of Shantou University Medical College, Shantou, China; ${ }^{5}$ Department of Thoracic Surgery, Shanghai Chest Hospital, Shanghai Jiaotong University, Shanghai, China; ${ }^{6}$ Department of Thoracic Surgery, Tianjin Medical University Cancer Hospital, Tianjin, China; ${ }^{7}$ Department of Thoracic Surgery, Zhejiang Cancer Hospital, Hangzhou, China; ${ }^{8}$ Department of Thoracic Surgery, Fudan University Shanghai Cancer Center, Shanghai, China; ${ }^{9}$ Department of Thoracic Surgery, Sichuan Cancer Hospital, Chengdu, China; ${ }^{10}$ Department of Thoracic Surgery, the University of Hong Kong-Shenzhen Hospital, Shenzhen, China; ${ }^{11}$ Department of Radiotherapy, Taizhou Hospital of Zhejiang Province Affiliated to Wenzhou Medical University, Linhai, China; ${ }^{12}$ Department of Thoracic Surgery, St. James's University Hospital, Bexley Wing, Leeds, UK; ${ }^{13}$ Department of Cardiothoracic Surgery, New York University Langone Health, New York, NY, USA; ${ }^{14}$ Department of Thoracic Surgery and Diseases of Esophagus, Aix-Marseille University, Hôpital Nord, Chemin des Bourrely, Marseille Cedex, France; ${ }^{15}$ Department of Surgery, Inova Fairfax Medical Campus, Falls Church, VA, USA; ${ }^{16}$ University Cancer Center Leipzig, University Medicine Leipzig, Leipzig, Germany

Contributions: (I) Conception and design: B Chen, C Zhu; (II) Administrative support: D Ma, M Ye, B Chen, C Zhu; (III) Provision of study materials or patients: All authors; (IV) Collection and assembly of data: J Shen, M Kong, H Yang, K Jin, Y Chen, W Fang; (V) Data analysis and interpretation: J Shen, M Kong, H Yang, K Jin, Y Chen, W Fang, Z Yu, W Mao, J Xiang, Y Han, Z Chen, H Yang, J Wang, Q Pang, X Zheng, H Yang, T Li, X Zhang, Q Li, G Wang, T Mao, X Guo, T Lin, M Liu, D Ma, M Ye, C Wang, Z Wang; (VI) Manuscript writing: All authors; (VII) Final approval of manuscript: All authors.

\#These authors contributed equally to this work.

*These authors contributed equally to this work as senior authors.

Correspondence to: Mr. Chengchu Zhu; Mr. Baofu Chen. Key Laboratory of Minimally Invasive Techniques \& Rapid Rehabilitation of Digestive System Tumor of Zhejiang Province, Linhai, China; Department of Cardiothoracic Surgery, Taizhou Hospital of Zhejiang Province Affiliated to Wenzhou Medical University, 150\# Ximen Street, Linhai 317000, China. Email: zhucc@enzemed.com; chenbf@enzemed.com.

\begin{abstract}
Background: Few studies have exclusively investigated the value of pathological complete response (pCR), in esophageal squamous cell carcinoma (ESCC) patients, although it is a clinically significant parameter to evaluate the impact of neoadjuvant chemoradiotherapy (nCRT) on treatment outcome after surgery. The aim of our study was to explore the relationship between pCR after nCRT and survival among patients with local ESCC.

Methods: All patients receiving nCRT followed by surgery in NEOCRTEC5010-trial (NCT01216527) were included. Non-pCR patients were classified into three subgroups: ypTanyN0M0, ypT0NanyM0 and ypTanyNanyM0. The Kaplan-Meier method with log-rank test was employed to evaluate diseasefree survival (DFS) and overall survival (OS). Multivariate regression analysis was performed using a Cox proportional hazards model to identify clinicopathological parameters associated with pCR.
\end{abstract}

(c) Annals of Translational Medicine. All rights reserved. 
Results: Among the 185 patients included, 80 (43.2\%) achieved pCR after nCRT. The mean survival time of the pCR group was significantly longer than that of the non-pCR group (92.6 vs. 69.2 months; HR, 2.70; 95\% CI: $1.48-4.92 ; \mathrm{P}=0.001)$. The 5-year OS and DFS of the pCR group were $79.3 \%$ and $77 \%$ respectively, compared to $54.8 \%$ and $51.2 \%$, respectively, in the non-pCR group. The results showed that the OS and DFS of the ypTanyN0M0 group were better than those of the ypT0NanyM0 group and the yp TanyNanyM0 group. We also found that the number of dissected lymph nodes and pCR were independent risk factors for DFS and OS rates.

Conclusions: pCR after nCRT is an important prognostic indicator of OS and DFS in patients with ESCC. In addition, lymph-node status could represent an important parameter in the prognostic evaluation of esophageal cancer patients.

Keywords: Pathological response; neoadjuvant chemoradiotherapy (nCRT); surgery; survival; esophageal squamous cell cancer (ESCC)

Submitted Jun 28, 2021. Accepted for publication Aug 17, 2021.

doi: $10.21037 / \mathrm{atm}-21-3331$

View this article at: https://dx.doi.org/10.21037/atm-21-3331

\section{Introduction}

Esophageal cancer (EC) is a common type of malignant carcinoma with poor prognosis. Esophageal squamous cell carcinoma (ESCC) and esophageal adenocarcinoma (EADC) are two common histological subtypes of EC (1). Neoadjuvant chemoradiotherapy (nCRT) to improve the survival outcome of esophageal cancer treatment has been a standard method for locally advanced esophageal cancer in many regions $(2,3)$. Survival benefit from nCRT has been reported in some meta-analyses $(2,4,5)$, and randomized controlled trials (RCT) comparing nCRT plus surgery versus surgery alone have also produced consistent results in modern studies $(6,7)$.

The criticism against the trials that failed to demonstrate the superiority of the addition of nCRT mainly includes the heterogeneity of the patient populations and the nonstratification of survival by the pathological complete response (pCR) to nCRT. pCR is a clinically significant parameter in evaluating the impact of nCRT on treatment outcome after surgery. This parameter can assist in assessing tumor sensitivity to nCRT, predicting survival outcome and patterns of disease recurrence, and further optimizing treatment decisions (8). Among patients with EC, the patients achieving pCR had prolonged survival, whereas non-responders to nCRT had an even poorer prognosis than those receiving surgery alone $(9,10)$. However, there are very few studies that exclusively investigate ESCC and evidence for the value of pCR in this subtype of $\mathrm{EC}$ is therefore scarce.

The NEOCRTEC5010 trial was a well-designed, largescale RCT, that compared nCRT plus surgery versus surgery alone among 451 locally advanced EC patients. In contrast with previous RCTs in which EADC was predominant, the enrolled patients in the NEOCRTEC5010 trial were all ESCC patients (7). The results of the NEOCRTEC5010 trial indicated the superiority of multimodality therapy with respect to survival.

To better evaluate and understand the impact of nCRT on treatment outcome after surgery, specifically among the ESCC patient population, we analyzed the association between pCR after nCRT and overall survival (OS) and the disease-free survival (DFS) of the patients receiving nCRT plus surgery in the NEOCRTEC5010 trial.

We present the following article in accordance with the STROBE reporting checklist (available at https://dx.doi. org/10.21037/atm-21-3331).

\section{Methods}

\section{Patients}

The patient population of the present study consisted of all the patients who had received nCRT followed by surgery in the NEOCRTEC5010 trial (June 2007 to December 2014) (Figure 1). 


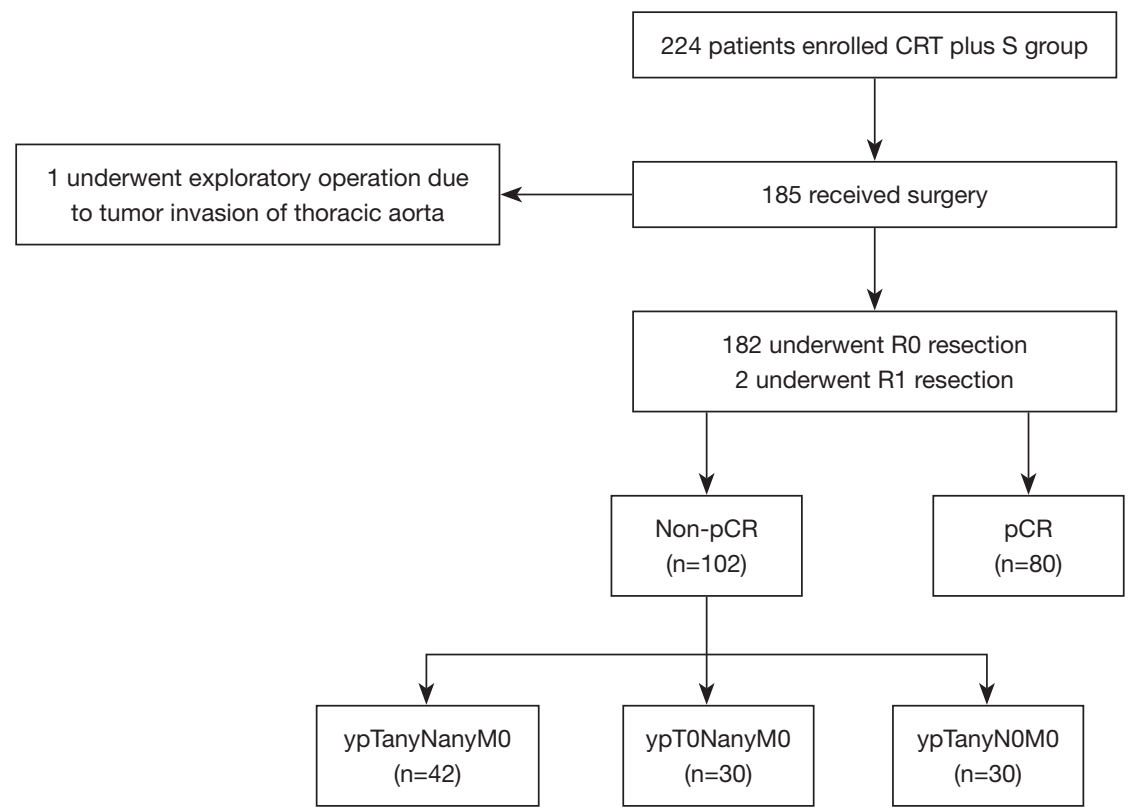

Figure 1 Schematic diagram of study design. CRT, chemoradiotherapy; ypTanyN0M0, lymph node complete response; pCR, pathological complete response; ypT0NanyM0, primary tumor complete response; S, surgery.

All patients met the following criteria: histologically diagnosed with potentially resectable thoracic ESCC with clinical stage IIB or III (T1-4N1M0/T4N0M0) before treatment; aged between 18 and 70 years; normal hematologic, renal and hepatic function; and a Karnofsky performance score $\geq 90$. Patients were excluded if they had a history of other malignancies; or were not suitable for surgery or reconstruction with a stomach conduit. A preoperative positive lymph node was defined as a diameter $\geq 1 \mathrm{~cm}$, which was measured by outcomes of computed tomography (CT), endoscopic ultrasound (EUS) or positron emission tomography (PET)-CT. This study was approved by the ethics committee of Taizhou Hospital of Zhejiang Province Affiliated to Wenzhou Medical University and the ethics committee of Sun Yatsen University Cancer Center (No. YP2007031). All procedures performed in this study involving human participants were in accordance with the Declaration of Helsinki (as revised in 2013). Individual consent for this retrospective analysis was waived.

\section{Pretreatment workup and staging}

Pretreatment staging included plain and contrastenhanced CT of the neck, thorax and abdomen, esophagogastroduodenoscopy with EUS, and cervical ultrasonography. All patients had undergone these tests. Tumor infiltration into the trachea or bronchial tree was excluded by bronchoscopy. PET and radionuclide bone imaging were optional.

\section{Preoperative chemotherapy}

The chemotherapy regimen consisted of $25 \mathrm{mg} / \mathrm{m}^{2}$ vinorelbine via intravenous (IV) bolus on days 1 and 8 and $75 \mathrm{mg} / \mathrm{m}^{2}$ cisplatin IV within 3 hours on day 1 or $25 \mathrm{mg} / \mathrm{m}^{2}$, cisplatin IV within 2 hours on days 1 to 4 administered every 3 weeks for two cycles. When the absolute neutrophil count $>1.5 \times 10^{9} / \mathrm{L}$ and the platelet count was $\geq 75 \times 10^{9} / \mathrm{L}$, a full dose of chemotherapy was administered. Otherwise, chemotherapy was delayed for up to 2 weeks until the counts recovered. When hematologic toxicity persisted for 2 weeks or longer, chemotherapy was discontinued.

\section{Preoperative radiotherapy}

A total dose of 40.0 Gy was administered in 20 fractions of 2.0 Gy, with 5 fractions per week, starting on the first day of the first cycle of chemotherapy. All patients were treated with external beam radiation, using the three-dimensional conformal radiation technique. The gross tumor volume 
was defined by the primary tumor and any enlarged regional lymph nodes. The clinical target volume provided a proximal and distal margin of $3 \mathrm{~cm}$ and a radial margin of 0.5 to $1.0 \mathrm{~cm}$ around the gross tumor volume. The planning target volume provided an $8-\mathrm{mm}$ margin of the clinical target volume.

\section{Surgery}

Surgery was performed 4 to 6 weeks after chemoradiotherapy. Surgery consisted of McKeown or Ivor Lewis esophagectomy, including two-field lymphadenectomy with total mediastinal lymph node dissection. Dissection of the left and right recurrent laryngeal nerve nodes was considered mandatory.

\section{Pathological analysis and definition}

Pathological examination reports contained the tumor type and extension, proximal and distal resection margins, tumor regression grade (Mandard score) and lymph nodes in the NEOCRTEC5010 trial. According to the results of pathological examination, these patients were characterized into two groups:

(I) pCR was defined as no evidence of residual tumor cells in the primary site and resected lymph nodes of the operative specimens;

(II) Non-pCR was defined as pCR not being achieved. The non-pCR patients could be further classified into three subgroups:

(i) ypTanyN0M0 referred to the category of nonpCR in which no residual tumor existed in the resected lymph nodes;

(ii) ypT0NanyM0 referred to the category of non-pCR in which no residual tumor existed in the primary site;

(iii) ypTanyNanyM0 was defined as the remainder of the non-pCRs other than ypTanyNOM0 and ypT0NanyM0.

\section{Outcomes and follow-up}

Overall survival was calculated as the time from the date of group assignment to the date of death or the last followup. DFS was calculated as the time from the date of R0 resection to the date of disease recurrence or death. Posttreatment follow-up was performed in the study centers once every 3 months within the first year and thereafter every 6 months until death or the end of the study.

\section{Statistical analysis}

All patients who had received both nCRT and surgery were included in the evaluation of OS and pathological response. Only the patients who achieved R0 resection were included in the evaluation of DFS. Continuous variables were presented as the mean \pm SD. Frequency counts and proportions were adopted to describe the category variables. Continuous variables were compared using the $t$ test or the nonparametric Mann-Whitney $\mathrm{U}$ test as indicated. Chi-square tests or Fisher's exact tests were used to make comparisons between groups. The optimal cutoff values for lymph nodes examined were determined using a timedependent receiver operating curve (ROC) analysis with Cutoff Finder (http://molpath.charite.de/cutoff/). The lymph-node-examined cutoff point of 19 was used to stratify the patients.

For survival analyses, the Kaplan-Meier method with log-rank test was used to analyze the correlation between pathological response and OS (and DFS). We used the Cox proportional hazards model to calculate HRs and 95\% CIs. Statistical analyses were performed using SPSS 20.0. Figures were formulated by $\mathrm{R}$ software with the survminer package. $\mathrm{P}<0.05$ was considered to be statistically significant.

\section{Results}

\section{Clinical characteristics}

A total of 185 patients who received nCRT followed by surgery in the NEOCRTEC5010 trial were included in the present study. A schematic diagram of patient inclusion and exclusion in the present study is shown in Figure 1. Clinical characteristics comparing patients with pCR to non-pCR are presented in Table 1. No baseline factors were associated with pCR. Pathological responses were assessed in all patients. Of the 185 patients, 80 patients achieved pCR (43.2\%), and 182 patients achieved R0 resection. Among the 105 non-pCR patients (56.8\%), 30 patients achieved ypTanyN0M0, 30 patients achieved ypT0NanyM0, and 42 patients were categorized as ypTanyNanyM0. None of the clinicopathological parameters analyzed was significantly associated with pCR. 
Table 1 Clinical characteristics of study population and factors associated with pathological complete response

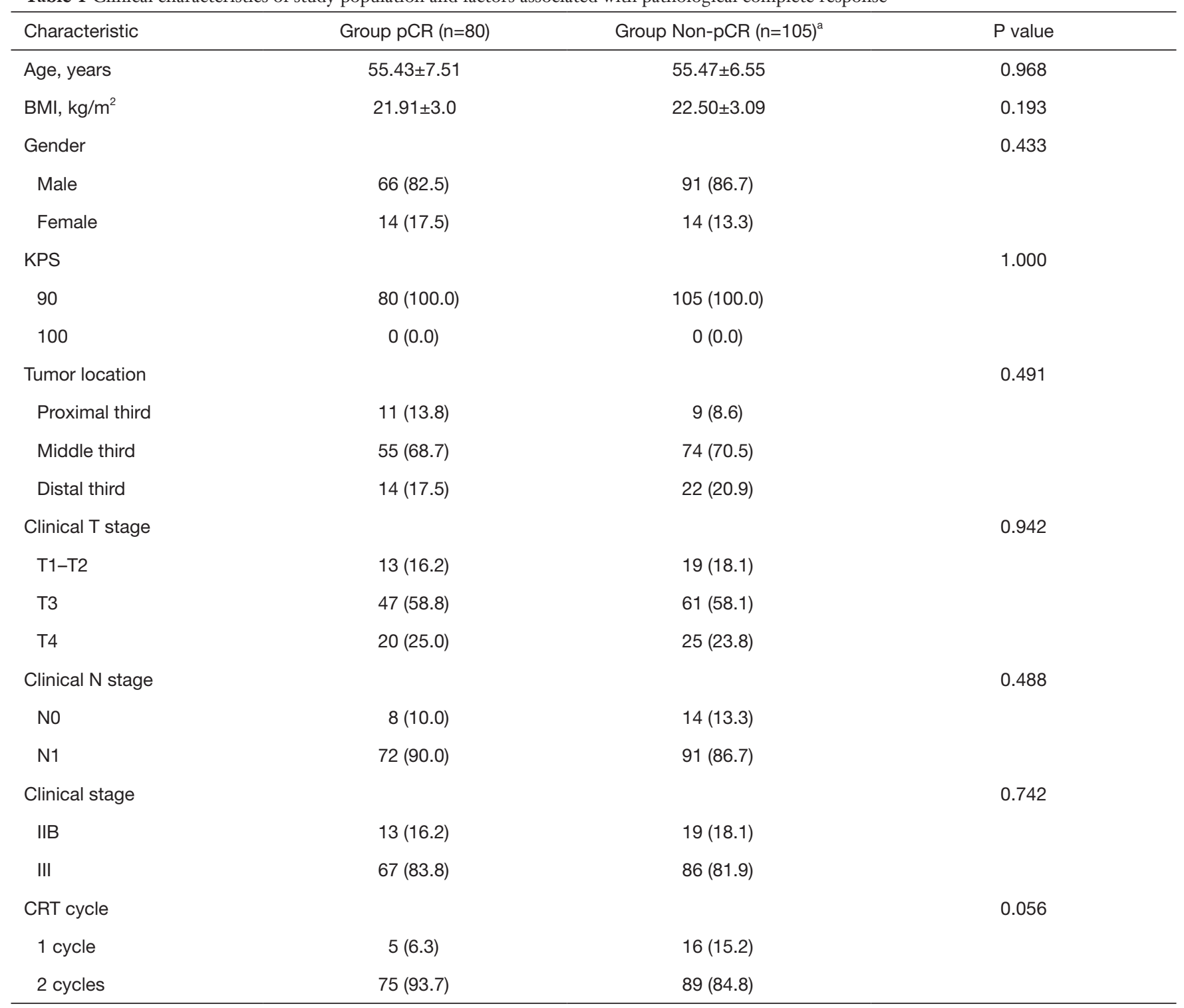

a, group non-pCR included 30 patients achieved ypTanyNOMO, 30 patients achieved ypTONanyM0, and 42 patients categorized as ypTanyNanyMO. BMI, body mass index; CRT, chemoradiotherapy; KPS, Karnofsky Performance Score; pCR, pathological complete response.

\section{The OS and DFS of the patients in the pCR group and the non-pCR group}

The median follow-up time was 43.6 months (IQR, 26.461.1 months) in the entire cohort. The mean OS time for the entire cohort was 79.8 months (95\% CI: 72.7-87.0). In the Kaplan-Meier analysis, the mean OS time of the patients with pCR was 92.6 months (95\% CI: 84.3-100.8), which was significantly improved compared with the 69.2-month mean (95\% CI: 59.7-78.8) in the patients with
non-pCR. Multivariate Cox proportional hazards regression analysis indicated that $\mathrm{pCR}$ predicted for death (HR, 2.70; 95\% CI: 1.48-4.92; $\mathrm{P}=0.001$; Figure $2 A$ ). The OS rate was $95 \%$ in patients achieving pCR at 1 year, $86.2 \%$ at 3 years, and $79.3 \%$ at 5 years. Among the 182 patients who achieved R0 resection, the mean DFS was 70.5 months $(95 \% \mathrm{CI}$ : 63.1-77.7). The mean DFS time of the pCR group was 85 months (95\% CI: 74.9-95.2), which was significantly longer than the 59.4-month mean (95\% CI: 49.8-69.0) 


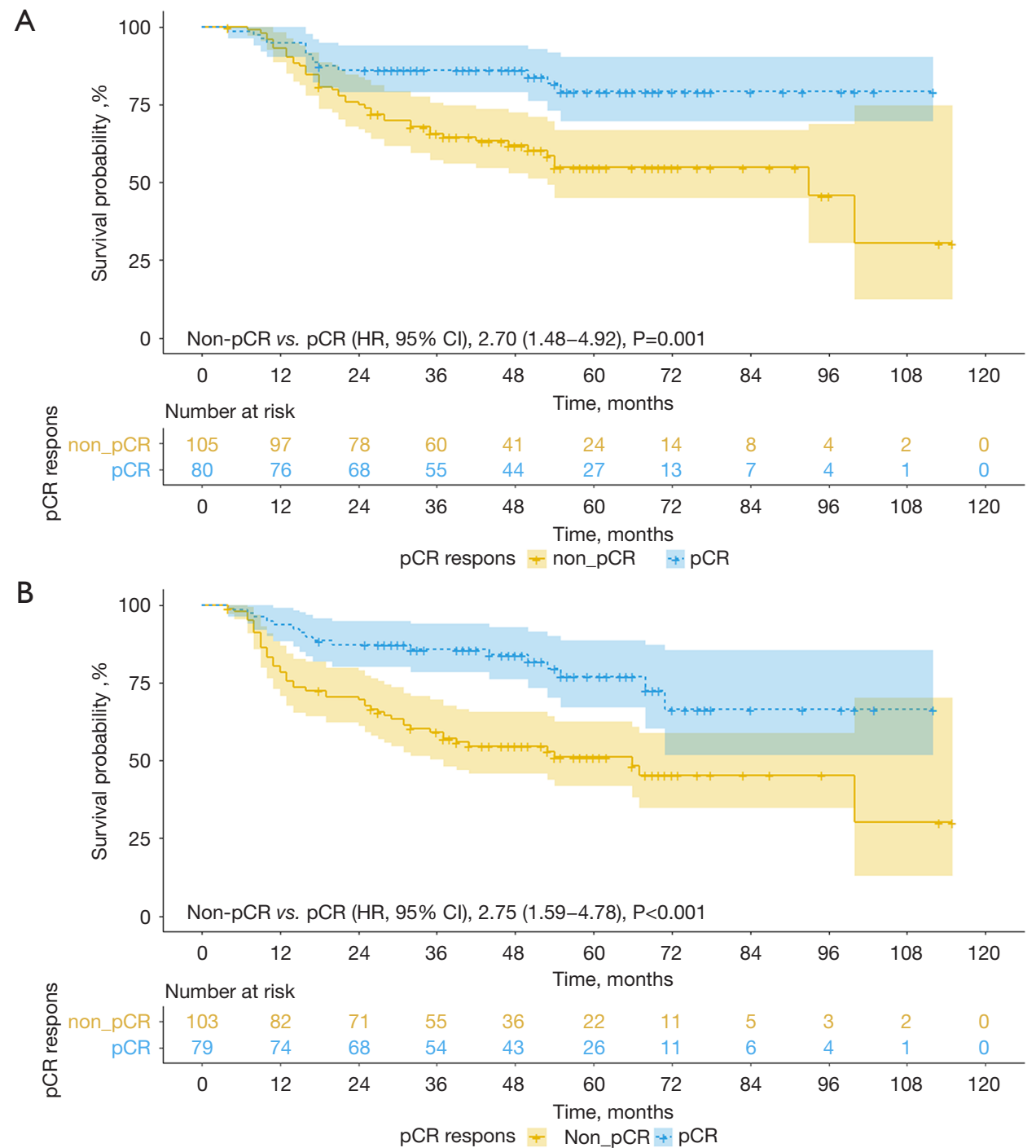

Figure 2 OS (A) and DFS (B) in the patients achieving pCR and non-pCR. OS, overall survival; DFS, disease-free survival; pCR, pathological complete response.

found in the non-pCR group. In the multivariable Cox regression models, $\mathrm{pCR}$ was an independent risk factor for DFS (HR, 2.75; 95\% CI: 1.59-4.78; $\mathrm{P}<0.001$; Figure 2B). The 5 -year DFS rate in the pCR group was $77 \%$.

\section{The OS and DFS in the non-pCR patients}

Among the non-pCR patients, the patients achieving ypTanyN0M0 had a mean OS time of 90.3 months $(95 \%$ CI: 77.3-103.4), in comparison with 56.7 months (95\% CI: 43.4-70.1) in the patients achieving ypT0NanyM0 and 57.3 months (95\% CI: 42.7-71.9) in the patients achieving
ypTanyNanyM0 ( $\mathrm{P}=0.001$; Figure $3 A)$. Furthermore, the mean OS time of the ypTanyNOM0 group was significantly greater than that of the ypT0NanyM0 group $(\mathrm{P}=0.036)$, whereas no significant difference was noted between the ypT0NanyM0 and ypTanyNanyM0 groups $(\mathrm{P}=0.330)$. The OS rate in the ypTanyN0M0 group was $100 \%$ at 1 year, $93.5 \%$ at 3 years, and $76.6 \%$ at 5 years. DFS was also compared among the 3 non-pCR groups. The mean DFS time was 83 months (95\% CI: 69.2-96.9) in the ypTanyN0M0 group, 38.2 months (95\% CI: 28.7-47.7) in the ypT0NanyM0 group, and 46.3 (95\% CI: 30.9-61.8) in the yp TanyNanyM0 group $(\mathrm{P}<0.001$; Figure $3 B)$. 

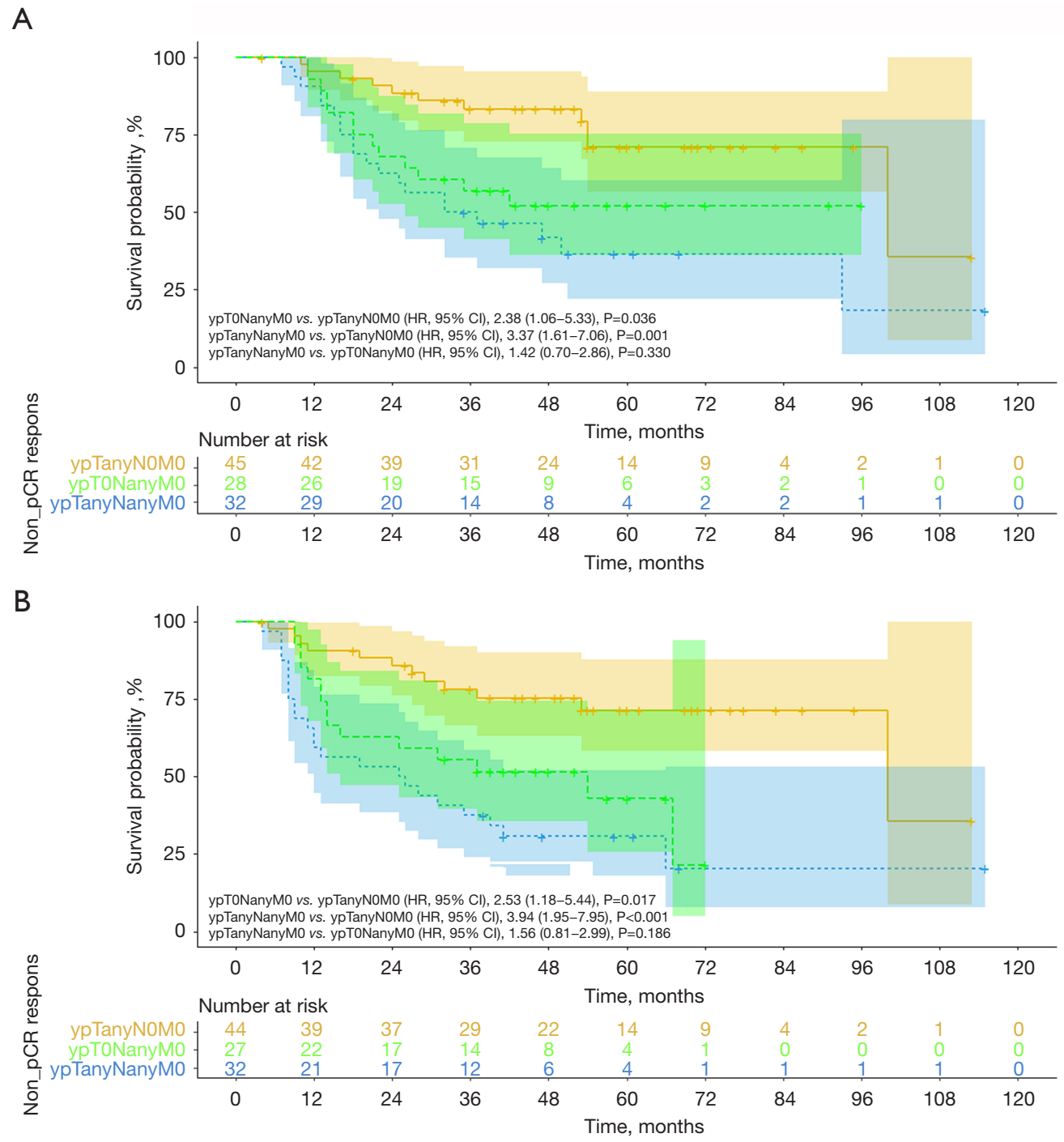

Figure 3 OS (A) and DFS (B) in the non-pCR patients achieving ypTanyN0M0, ypT0NanyM0 and ypTanyNanyM0. ypTanyN0M0, lymph node pathological complete response; ypT0NanyM0, primary tumor pathological complete response; OS, overall survival; DFS, disease-free survival; pCR, pathological complete response.

Furthermore, there are no significant differences of OS $(\mathrm{P}=0.93$; Figure $4 A)$ and $\mathrm{DFS}(\mathrm{P}=0.91$; Figure $4 B)$ rates between the ypTanyN0M0 and pCR patients.

\section{Factors associated with OS and DFS}

The univariate analysis indicated that both pCR and 19 or more lymph nodes examined were prognostic indicators for OS $(\mathrm{P}=0.001$ and $\mathrm{P}=0.003)$ and $\mathrm{DFS}(\mathrm{P}<0.001$ and $\mathrm{P}=0.008)$. After adjusting for significant variables in the multivariate analysis, pathological response, and the number of nodes examined were independent predictors of both OS and DFS (both $\mathrm{P}<0.05$ ). The other clinicopathological parameters analyzed did not significantly affect OS or DFS (Tables 2,3).

\section{Discussion}

The current study indicated that the number of dissected lymph nodes and $\mathrm{pCR}$ were independent predictive factors of survival for ESCC patients who underwent nCRT plus surgery. Furthermore, lymph node status was significantly associated with outcomes of OS and DFS. This is a second 
A
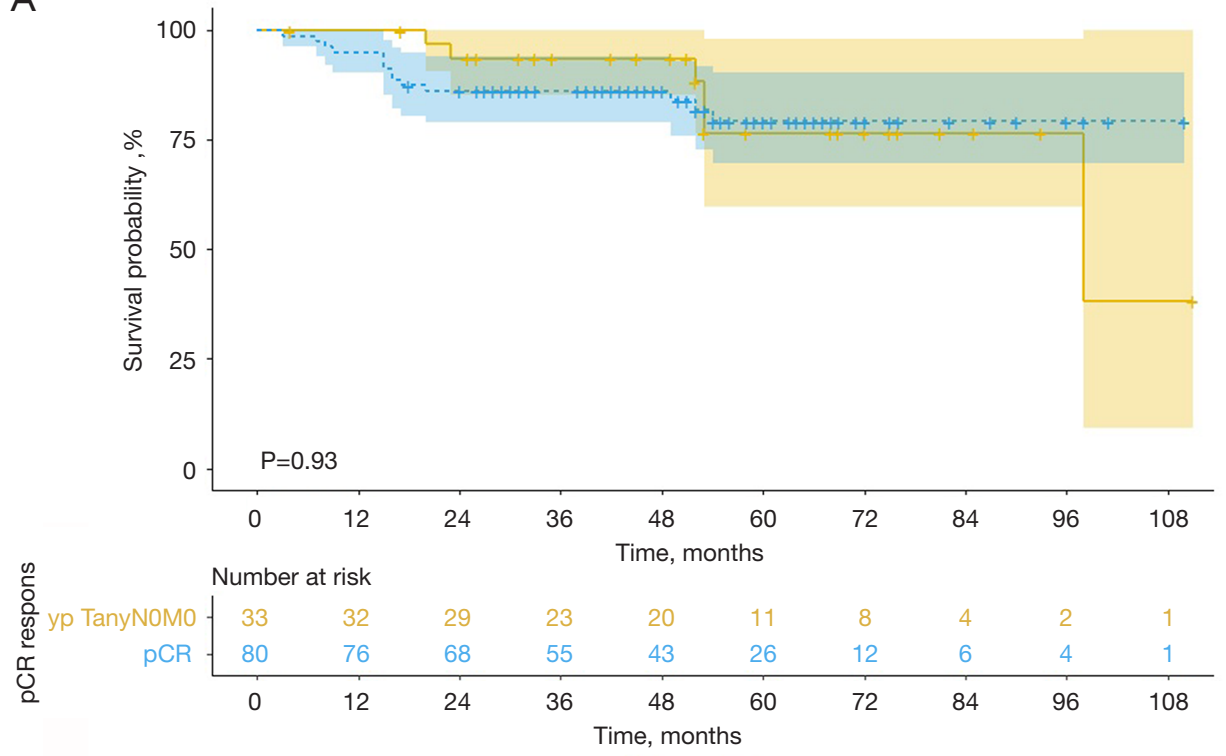

yp TanyNOMO + pCR + -

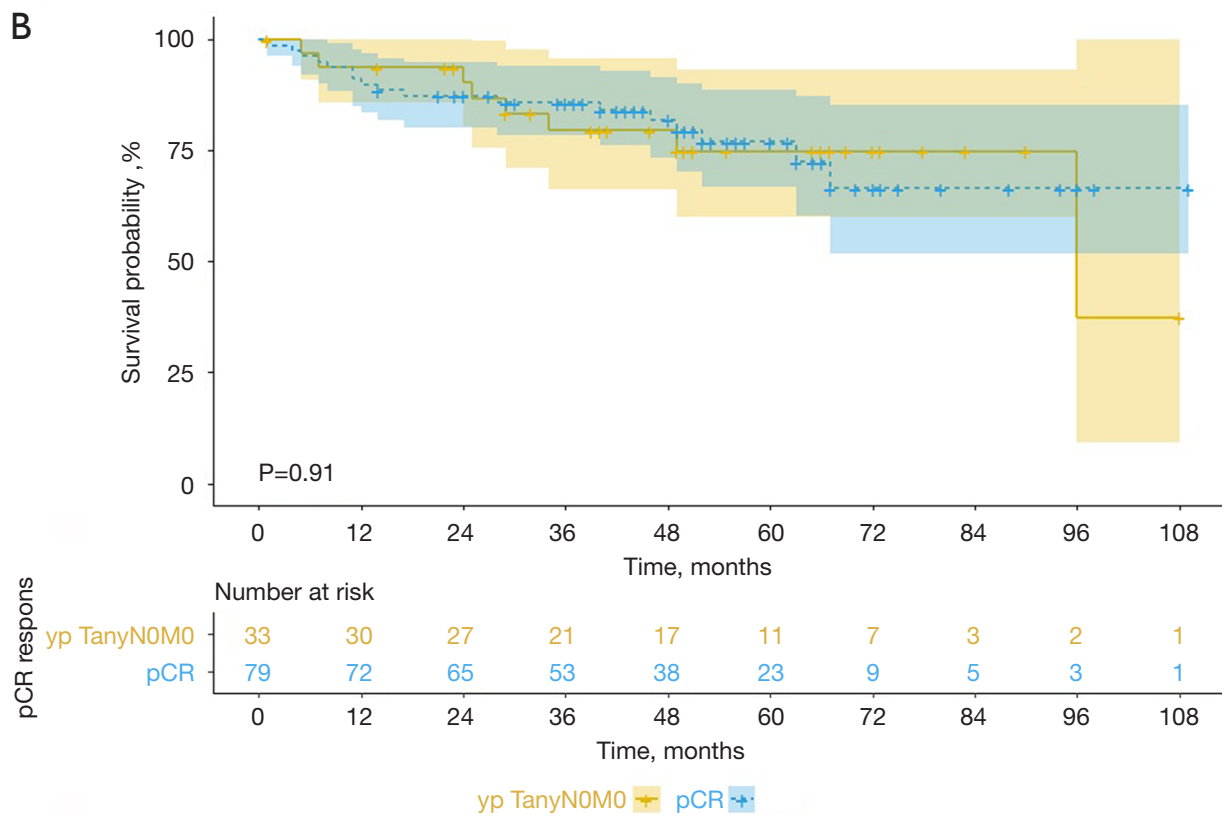

Figure 4 OS (A) and DFS (B) in the patients achieving pCR and ypTanyN0M0. OS, overall survival; DFS, disease-free survival; ypTanyN0M0, lymph node pathological complete response; pCR, pathological complete response.

finding from a well-designed and large-scale RCT named NEOCRTEC5010 trial, which compared nCRT plus surgery versus surgery alone among 451 locally advanced EC patients.

The current findings showed that 80 patients (43.2\%) achieved pCR among the included 185 ESCC patients. Some studies have analyzed the pCR rate among the included ESCC patients. In a retrospective Chinese study among 392 ESCC patients, a pCR rate of $25.8 \%$ was achieved after surgery (11). In the European CROSS trial, the pCR rate in 37 ESCC patients with a WHO performance score of 2 or greater reached $49 \%$ (6). A pCR rate of $41.2 \%$ was achieved 
Table 2 Univariate and multivariable analysis for overall survival in relation to clinicopathological parameters

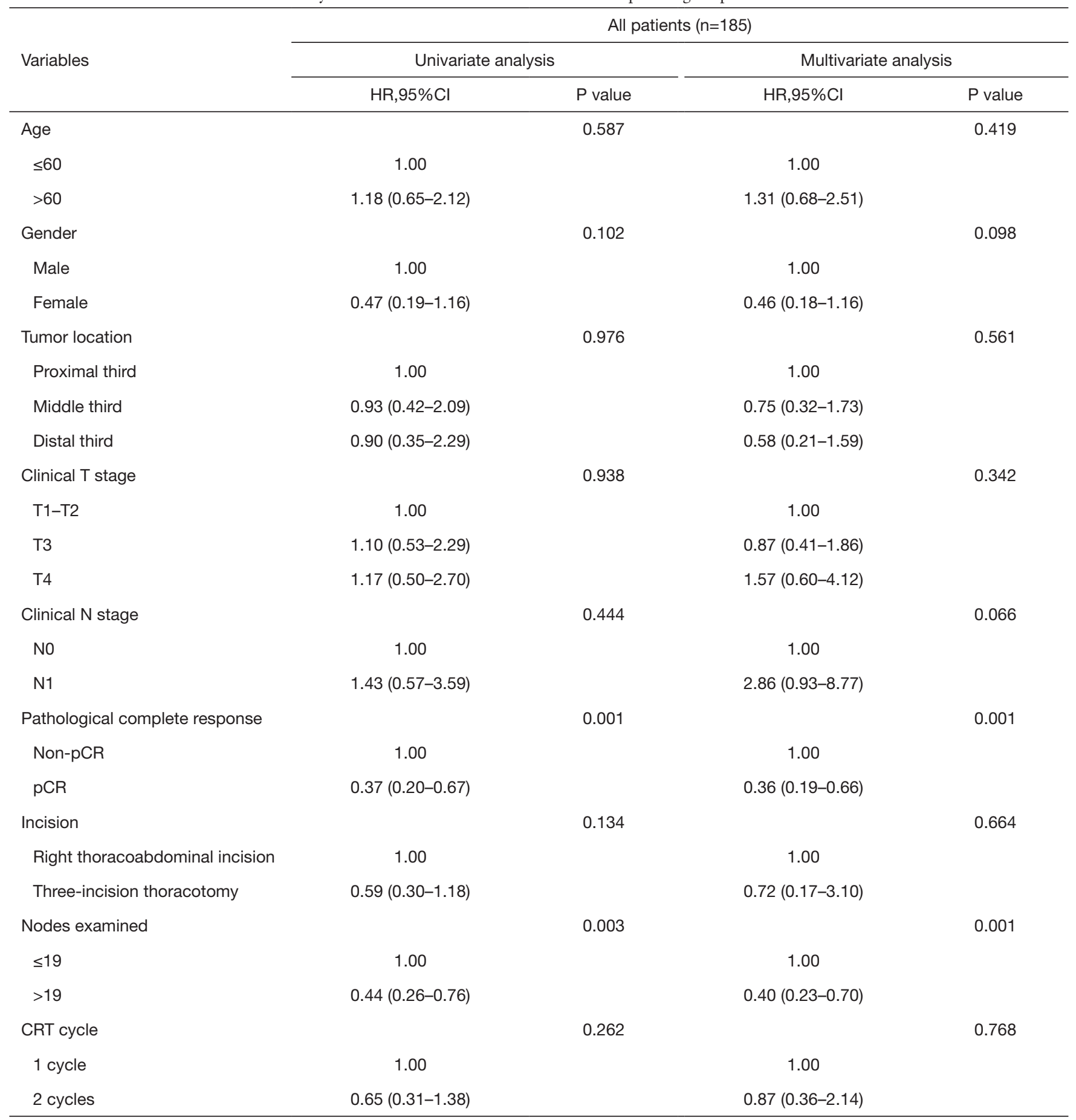

$\mathrm{BMI}$, body mass index; Cl, confidence interval; CRT, chemoradiotherapy; HR, hazard ratio; pCR, pathological complete response.

among a total of 34 ESCC patients in the North American study (8). The pCR rate of our study was comparable to that in these two aforementioned studies, and considerably increased compared with that found in the retrospective study with a relatively large number of ESCC patients.

In our study, the pCR group obtained a significantly 
Table 3 Univariate and multivariate analysis for disease-free survival in relation to clinicopathological parameters

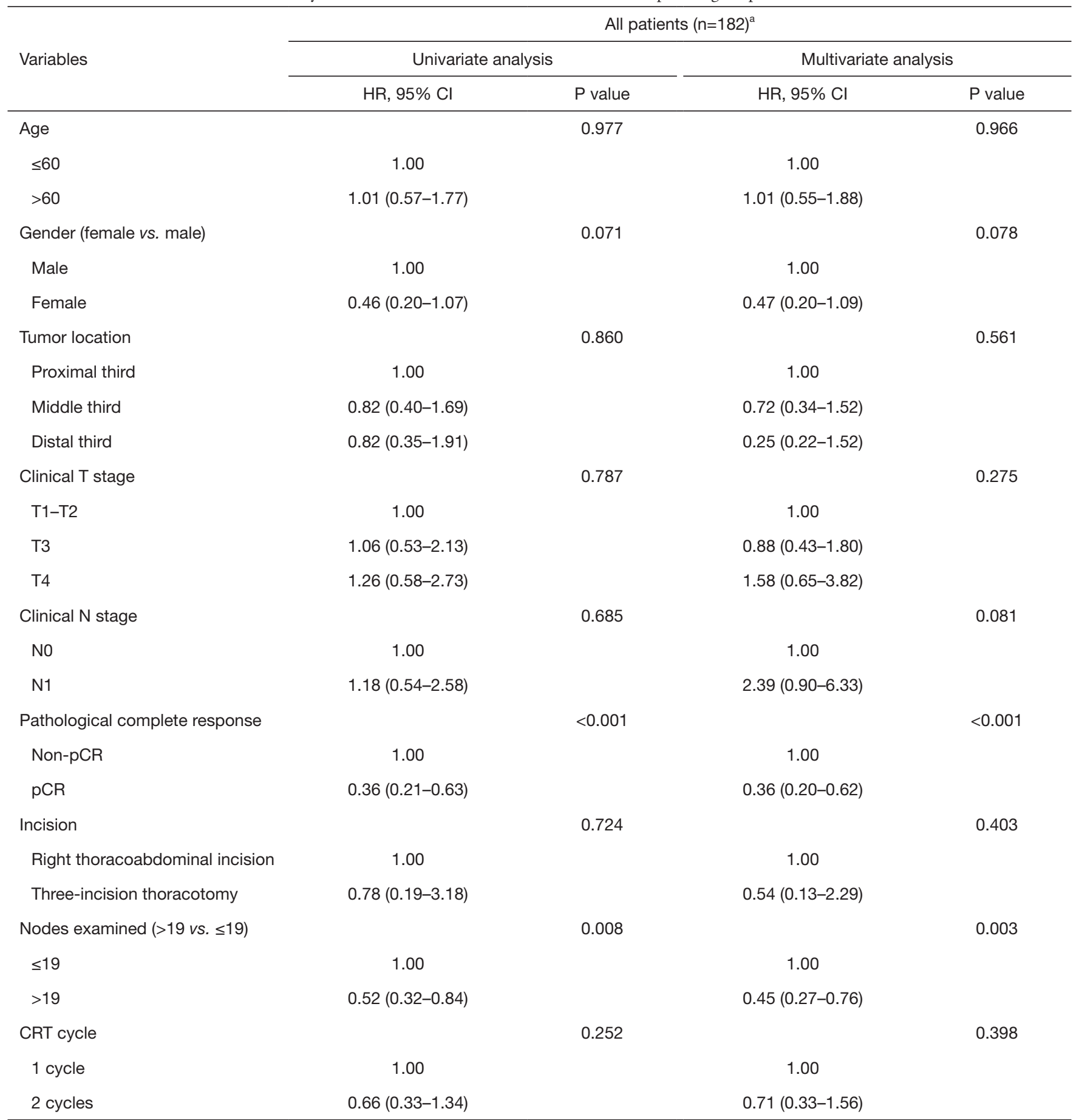

${ }^{\mathrm{a}}$, three patients who did not achieve RO resection were not included in the analysis. BMI, body mass index; $\mathrm{Cl}$, confidence interval; CRT, chemoradiotherapy; $\mathrm{HR}$, hazard ratio; $\mathrm{PCR}$, pathological complete response.

longer OS of 92.6 months and a higher 5-year OS rate of $79.3 \%$, which were significantly better than those of the non-pCR group. The 5-year OS rate in the pCR group was better than the result reported previously, in which the 5 -year OS rate ranged mainly between $47 \%$ and $55 \%(6,7,9,10)$. The difference might be due to the higher OS and 5 -year 
OS rate obtained in the NEOCRTEC5010 trial, which had been attributed to the implementation of a total mediastinal lymph node dissection, especially a recurrent laryngeal nerve node dissection, and the extensive clinical experience of the EC treatment in East Asian centers, as described in the article reporting the results of the NEOCRTEC5010 trial (7). This notion was also confirmed in the study by Visser et al. that recruited 2,698 patients who underwent nCRT followed by esophagectomy between 2005 and 2014 from the Netherlands Cancer Registry (12). The study indicated that a high lymph node yield was associated with improved overall survival. A possible explanation for the extension of overall survival could be the removal of micrometastases $(13,14)$.

The number of dissected lymph nodes was another independent risk factor for OS and DFS in our study. Several studies have shown that the number of lymph nodes removed was an independent predictor of survival for patients with esophageal cancer who underwent esophagectomy $(15,16)$. To maximize survival, Groth et al. suggested that esophageal cancer patients should have at least 30 lymph nodes examined pathologically (17). In our study, patients received nCRT plus esophagectomy, and we suggest that at least 19 lymph nodes should be examined pathologically. However, a retrospective study of 358 resected esophageal cancer patients after nCRT demonstrated that the number of lymph nodes harvested during esophagectomy did not impact survival (18). The chemotherapy regimens included cisplatin and bolus 5 -fluorouracil (5-FU), cisplatin and protracted infusion 5-FU, carboplatin and paclitaxel, and oxaliplatin and protracted infusion $5-\mathrm{FU}$. The median radiation dose was 50.4 (range, 45-59.4) Gy with an unknown radiotherapy cycle. With such differences from our study, future research will be needed to investigate the relationship between the number of lymph nodes removed and survival outcome in ESCC or EADC patients who underwent esophagectomy after different chemoradiotherapy regimens.

Some previous studies have demonstrated that apart from pCR patients, those achieving partial pathological response might also achieve superior survival $(8,19)$. Thus, it is necessary to stratify non-pCR patients to further identify the patients who have a possibility of survival improvement, instead of relying on pCR alone. In the present study, the OS and DFS of the non-pCR patients achieving ypTanyNOM0 were in the same range as the pCR patients, and were significantly increased compared with those in the non-pCR group with ypT0NanyM0 and the ypTanyNanyM0 group. This finding is consistent with the results of previous studies, in which pathological lymph-node-negative patients showed prolonged survival compared with lymph-node-positive patients (20). These results indicate that among the non-pCR patients, a portion of patients, namely those who achieve ypTanyN0M0, can still obtain a favorable survival outcome.

Some limitations in the present study should be noted. First, this is a retrospective study, although the data analyzed were collected prospectively. Second, the data were obtained from several centers, and no central histopathological assessment was performed, which potentially resulted in bias arising from the difference in reporting and assessment.

\section{Conclusions}

Our study provides evidence that pCR after nCRT is an important prognostic indicator of OS and DFS in patients with locally advanced esophageal squamous cell cancer. In addition, lymph-node status rather the primary tumor response could exhibit great importance in the prognostic evaluation of EC patients.

\section{Acknowledgments}

Funding: Sun Yat-sen University Clinical Research 5010 Program (grant number: 2007048), Research Fund of the Taizhou Science and Technology Agency (grant number: 1701KY25).

\section{Footnote}

Reporting Checklist: The authors have completed the STROBE reporting checklist. Available at https://dx.doi. org/10.21037/atm-21-3331

Data Sharing Statement: Available at https://dx.doi. org/10.21037/atm-21-3331

Conflicts of Interest: All authors have completed the ICMJE uniform disclosure form (available at https://dx.doi. org/10.21037/atm-21-3331). The authors have no conflicts of interest to declare.

Ethical Statement: The authors are accountable for all aspects of the work in ensuring that questions related to the accuracy or integrity of any part of the work are appropriately investigated and resolved. This study was 
approved by the ethics committee of Taizhou Hospital of Zhejiang Province Affiliated to Wenzhou Medical University and the ethics committee of Sun Yat-sen University Cancer Center (No. YP2007031). All procedures performed in this study involving human participants were in accordance with the Declaration of Helsinki (as revised in 2013). Individual consent for this retrospective analysis was waived.

Open Access Statement: This is an Open Access article distributed in accordance with the Creative Commons Attribution-NonCommercial-NoDerivs 4.0 International License (CC BY-NC-ND 4.0), which permits the noncommercial replication and distribution of the article with the strict proviso that no changes or edits are made and the original work is properly cited (including links to both the formal publication through the relevant DOI and the license). See: https://creativecommons.org/licenses/by-nc-nd/4.0/.

\section{References}

1. Torre LA, Bray F, Siegel RL, et al. Global cancer statistics, 2012. CA Cancer J Clin 2015;65:87-108.

2. Sjoquist KM, Burmeister BH, Smithers BM, et al. Survival after neoadjuvant chemotherapy or chemoradiotherapy for resectable oesophageal carcinoma: an updated metaanalysis. Lancet Oncol 2011;12:681-92.

3. Yoshida N, Baba H. Neoadjuvant chemoradiotherapy followed by surgery is associated with better survival outcomes in patients with locally advanced esophageal squamous cell carcinoma. J Thorac Dis 2018;10:S3067-8.

4. Gebski V, Burmeister B, Smithers BM, et al. Survival benefits from neoadjuvant chemoradiotherapy or chemotherapy in oesophageal carcinoma: a meta-analysis. Lancet Oncol 2007;8:226-34.

5. Zhang CD, Zeng YJ, Li HW, et al. Neoadjuvant chemotherapy for nonmetastatic esophago-gastric adenocarcinomas: a systematic review and meta-analysis. Cancer Invest 2013;31:421-31.

6. Shapiro J, van Lanschot JJB, Hulshof MCCM, et al. Neoadjuvant chemoradiotherapy plus surgery versus surgery alone for oesophageal or junctional cancer (CROSS): long-term results of a randomised controlled trial. Lancet Oncol 2015;16:1090-8.

7. Yang H, Liu H, Chen Y, et al. Neoadjuvant Chemoradiotherapy Followed by Surgery Versus Surgery Alone for Locally Advanced Squamous Cell Carcinoma of the Esophagus (NEOCRTEC5010): A Phase III
Multicenter, Randomized, Open-Label Clinical Trial. J Clin Oncol 2018;36:2796-803.

8. Meredith KL, Weber JM, Turaga KK, et al. Pathologic response after neoadjuvant therapy is the major determinant of survival in patients with esophageal cancer. Ann Surg Oncol 2010;17:1159-67.

9. Berger AC, Farma J, Scott WJ, et al. Complete response to neoadjuvant chemoradiotherapy in esophageal carcinoma is associated with significantly improved survival. J Clin Oncol 2005;23:4330-7.

10. Dittrick GW, Weber JM, Shridhar R, et al. Pathologic nonresponders after neoadjuvant chemoradiation for esophageal cancer demonstrate no survival benefit compared with patients treated with primary esophagectomy. Ann Surg Oncol 2012;19:1678-84.

11. Chao YK, Chang HK, Tseng CK, et al. Development of a nomogram for the prediction of pathological complete response after neoadjuvant chemoradiotherapy in patients with esophageal squamous cell carcinoma. Dis Esophagus 2017;30:1-8.

12. Visser E, van Rossum PSN, Ruurda JP, et al. Impact of Lymph Node Yield on Overall Survival in Patients Treated With Neoadjuvant Chemoradiotherapy Followed by Esophagectomy for Cancer: A Population-based Cohort Study in the Netherlands. Ann Surg 2017;266:863-9.

13. Karstens KF, Ghadban T, Effenberger K, et al. Lymph Node and Bone Marrow Micrometastases Define the Prognosis of Patients with pN0 Esophageal Cancer. Cancers (Basel) 2020;12:588.

14. Yanagi M, Sasaki K, Uchikado Y, et al. Effect of Neoadjuvant Chemoradiotherapy on Lymph Node Micrometastases in Thoracic Esophageal Cancer. Anticancer Res 2018;38:893-900.

15. Peyre CG, Hagen JA, DeMeester SR, et al. Predicting systemic disease in patients with esophageal cancer after esophagectomy: a multinational study on the significance of the number of involved lymph nodes. Ann Surg 2008;248:979-85.

16. Rizk NP, Ishwaran H, Rice TW, et al. Optimum lymphadenectomy for esophageal cancer. Ann Surg 2010;251:46-50.

17. Groth SS, Virnig BA, Whitson BA, et al. Determination of the minimum number of lymph nodes to examine to maximize survival in patients with esophageal carcinoma: data from the Surveillance Epidemiology and End Results database. J Thorac Cardiovasc Surg 2010;139:612-20.

18. Shridhar R, Hoffe SE, Almhanna K, et al. Lymph node harvest in esophageal cancer after neoadjuvant 
chemoradiotherapy. Ann Surg Oncol 2013;20:3038-43.

19. Brücher BL, Becker K, Lordick F, et al. The clinical impact of histopathologic response assessment by residual tumor cell quantification in esophageal squamous cell carcinomas. Cancer 2006;106:2119-27.

Cite this article as: Shen J, Kong M, Yang H, Jin K, Chen Y, Fang W, Yu Z, Mao W, Xiang J, Han Y, Chen Z, Yang H, Wang J, Pang Q, Zheng X, Yang H, Li T, Zhang X, Li Q, Wang G, Mao T, Guo X, Lin T, Liu M, Ma D, Ye M, Wang C, Wang Z, Brunelli A, Cerfolio RJ, D’Journo XB, Fernando HC, Lordick F, Fu J, Chen B, Zhu C; written on behalf of the AME Thoracic Surgery Collaborative Group. Pathological complete response after neoadjuvant treatment determines survival in esophageal squamous cell carcinoma patients (NEOCRTEC5010). Ann Transl Med 2021;9(20):1516. doi:10.21037/atm-21-3331
20. Rizk NP, Venkatraman E, Bains MS, et al. American Joint Committee on Cancer staging system does not accurately predict survival in patients receiving multimodality therapy for esophageal adenocarcinoma. J Clin Oncol 2007;25:507-12. 